IIIi poélica 


\title{
JORNADA LABORAL DE UN POETA BARCELONÉS
}

\section{Francisco Ferrer Lerín}

\author{
A Don Antonio Chacón \\ que desde Colmenar Viejo \\ me ha mandado un requesón
}

Voy a recordar para todos ustedes, sucintamente, lo que era la jornada laboral de un poeta barcelonés en el periodo comprendido entre 1959 y 1974.

Por una cuestión de método vamos a agrupar las actividades en cinco apartados temáticos olvidándonos del momento en que podían desarrollarse; es decir, carecen de importancia elementos como mañana, tarde, noche o incluso otoño, invierno y primavera; el verano es cl veraneo por lo que no sólo cambia cl escenario -de la ciudad al campo- sino que el poeta descansa del ejercicio de sus funciones propias.

\section{Apartado 1. Incremento cultural.}

a) Ya Hitchcock comenta a Truffaut el desdén por el cine que manifiestan las clases acomodadas, o sea las clases cultas, ya que el intento de unir miseria a cultura es muy reciente y de difícil encaje. Se pronuncia en los siguientes términos (utilizo la traducción de Ramón G. Redondo para Alianza Editorial de Le cinema selon Hitchcock de François Truffaut): «Al principio de la historia del cine, este arte era extraordinariamente menospreciado por los intelectuales; también en Francia, pero ciertamente menos que en Inglaterra. Ningún inglés bien educado se habría dejado ver entrando en una sala de cine. Esto no se hacía. Usted sabe que en Inglaterra hay una gran conciencia de clase y de casta. Cuando la Paramount abrió el teatro Plazza en Londres, algunas personas de la buena sociedad empezaron a ir al cine; para ellas se dispusieron algunas butacas en los palcos cuyo precio era tan elevado que se les llamaba la fila de los millonarios». Pues bien, esta circunstancia prevalece en los años y en el lugar que nos ocupa. Sentir pasión por cl cine, incluso frecuentando, religiosamente, las pestilentes salas de reestreno, no forma parte de la construcción del personaje epataburgueses, sino de una convicción propia de buen salvaje, individuo íntegro que ve en el séptimo arte precisamente eso, la conjunción de literatura, imagen, música y magia. (Hubo días en que cayeron más de cuatro cintas.) 


\section{JORNADA LABORAL DE UN POETA BARCELONÉS}

b) La sagrada forma libro estaba destinada por su cualidad de objeto ligero y pequeño, ofertada en estantes y mesas, a su robo y colección. Nadie hurtó más y mejor que aquel grupo de poetas caminantes, merodeadores, sabuesos de impar olfato, bibliófilos a la carrera, conocedores de cada una de las librerías de nuevo y de viejo hasta extremos de delirio. Como se recuerda en los versos homenaje a Emilio Carrère «ahora hablemos de al acpa / o capa / que de todo hay / de esa pintoresca y corcusida / simbiótica melaza del no madrugar / espasmo que es vida / vida de la capa / y del que escapa / de esa tapa que es tapujo de infamias cometidas / y de ignotas adquisiciones», fueron los atuendos de cada época -americana, camisa y corbata entonces- los que conformaron los modos de sustracción y la filosofía diaria. Pero no sería justo olvidar aquellas grandes razzias, comunales, comarcales, motorizadas, como la del viernes veintiuno de junio de 1963 en la que participaron los dos grandes poetas del momento amén de dos criados -chófer y camelador-y que supuso el evento cultural más notable de la década: en la falda de la sagrada montaña de Monserrat, en una extensa finca agrícola propiedad de los marqueses de Curculló, apartada unos cien metros de la masía principal, la ermita de Sant Ferrer ha sido transformada en estudio-biblioteca por el heredero, joven de aficiones intelectuales y sodomíticas, primo hermano de uno de los dos poetas y que, ese día, se halla ausente. Camelador habla con los payeses, Chófer sitúa el auto, y los dos poetas desvalijan concienzudamente los anaqueles: los tres tomos de la Nueva Geografía Universal por Ernesto Granger editados por Espasa-Calpe entre 1928 y 1929; los Episodios Nacionales en la edición ilustrada, en diez tomos, de La Guirnalda (1882-1885); la Historia Universal de César Cantú (diez tomos, Librería de Garnier hermanos, París, 1869); y la fundamental Biblioteca de Autores Españoles (setenta tomos, Imprenta de Rivadeneyra, Madrid, 1846-1880) fueron algunas de las adquisiciones más notables.

Aquí tengo una joya. (El conferenciante muestra al público una pequeña agenda publicitaria Laboratorios Reunidos, de Madrid - de cubiertas plásticas marrones.) Están reseñadas las acciones del poeta desde el sábado nueve de febrero, hasta el sábado veintidós de junio, de 1963. Aquí está todo. Al azar. Martes 26 de Marzo: Sabio (es el nombre de otro poeta, quien le acompaña en el día de hoy); Formica (en clave, "robar" libros); Fenomenología (se supone que el tratado de Husserl); Argos (nombre de la librería visitada); Léonor Fini (un libro sobre la singular artista, editado por Jean-Jacques Pauvert, profusamente ilustrado, con texto de Marcel Brion); Consentido (mote de uso interno aplicado al dueño de una librería de viejo de la calle Muntaner que se encerraba en la trastienda en cuanto entrábamos y nos saludábamos); La condena, El castillo; Francesa (se refiere a la Librería Francesa de Las Ramblas); Quasimodo (probablemente la Obra completa, bilingüc, traducida por varios autores, finales de los cincuenta, Buenos Aires, Editorial Sur); Libretería (es una calle, no debía conocer el nombre del establecimiento); El proceso; Cascarrabias (mote de uso interno aplicado al dueño de una librería de viejo de la calle Aribau). Y así todos los días. Un duro, pero espléndido trabajo.

También (vuelve a ojear la agenda), se encuentran aquí los títulos de las películas vistas, sus valoraciones -siguiendo el sistema de Cahiers du cinéma que puntúa por estrellas y puntos negros-, los nombres de las salas $-\mathrm{y}$ compruebo con preocupación que en su totalidad han desaparecido-y los de las personas acompañantes. Por ejemplo el jueves catorce de febrero, en el cine Regina, asisto, con José Mari (no recuerdo quién sería ese cinéfilo), a la proyección del díptico El tigre de Esnapur-La tumba india, inigualable entrega de aventuras exóticas realizada por Fritz. Lang, en 1959, a su regreso a Europa tras la etapa norteamericana. El viernes veintinueve de marzo, en el cine Tuset, con "el Sabio", vemos La mosca, dirigida en 1958 por Kurt Neumann, con Vincent Price, Patricia Owens y Herbert Marshall como actores más conocidos, y que constituye el glorioso antecedente de "las moscas" de los ochenta. Y, para terminar, para no cansarles con este subproducto poco poético, el domingo doce de mayo, en el cine Alcázar, Duelo en la alta sierra, del 62, también con "el Sabio", el western mejor, el más puro de Sam Peckinpah. Es evidente que eran buenos tiempos para los amantes del espectáculo cinematográfico. 


\section{FRANCISCO FERRER LERÍN}

\section{Apartado 2. Incremento económico.}

Había que vivir. Pese al confortable colchón familiar era necesario el dinero: hasta veinticuatro faros llegó a ostentar el frontal del Seiscientos Abarth de Máiquel Bundó -un casi poeta- y, en mi caso, los gastos, que hoy se denominarían de representación, eran sumamente abultados. La solución: el juego: el póquer: una labor para la que estaba genéticamente bien dotado: mi abuelo Ivo, aunque médico de profesión, formó su inmensa biblioteca comprando, en sus viajes por medio mundo, a precios a veces desorbitados, los volúmenes más valiosos; y todo gracias a las partidas que organizaba mensualmente en su caserón de Hix, en la Cerdaña francesa, donde desplumaba regularmente al notario y al farmacéutico de Puigcerdá, al juez de paz y al comadrón de Font-Romeu y así a todo el subsector profesional de la comarca. En la agenda (se vuelve a mostrar) se refleja abundantemente esta actividad. Por ejemplo, aquí (leyendo), el domingo 21 de abril: timba en G.V. (se refiere a un piso desocupado de la Gran Vía de propiedad familiar utilizado como garito de juego y como luego se verá para otros nobles fines), José Luis, Félix y Leopoldo (los puntos), + 650 (el botín, en pesetas). Otra fecha, al azar, las partidas son muy frecuentes, (ojeando): miércoles 1 de mayo: timba en Casa Joaquina (un restaurante en el que se jugaba tras el cierre); Lujuria, Senil, Marrano, Buenconejo (los puntos), +30 (pobre resultado). Ahora recuerdo (pequeña pausa, mirando al cielo) una partida en mi casa, en la casa de mis padres de la Diagonal, un enorme piso de casi seiscientos metros, lleno de muebles y objetos de anticuario, una partida que montamos para que Máiquel Bundó pudiera pagar una abultada factura resultado de nuevas compras en aquella tienda de accesorios del automóvil y en la que jugamos aconchabados el propio Máiquel, Toni Mascaró (otro compañero de fatigas, otro casi poeta, condiscípulo de Pedro Gimferrer..., sí, él debió de ser quién nos presentó..., estoy hablando de 1960 o como mucho de 1961) y un servidor. Busqué un pichón. El pichón perfecto. Conocido por "Truchas" o "El truchas" era el representante para la ciudad de Barcelona de una importante piscifactoría pirenaica. Lo desplumamos. No fueron necesarias las malas artes. Era demasiado tonto. Al salir de mi habitación, con la oscuridad reinante (eran pocas las luces, y además difusas, eclesiales) y un grado de aturdimiento, por el volumen de las pérdidas, aún mayor que el habitual, se dio con la cabeza contra el filo de una puerta entreabierta, una de las muchas que había que atravesar, cayendo fulminado al suelo. Tardó en volver en sí. Parece como si lo estuviera viendo. Abrió los ojos, y empezó a repetir: « Buena jugada, buena jugada! ¡Dame dos cartas!». Ya de pic, e incluso entrando en el ascensor, seguía con la cantinela. Por cierto, ese caballero, era el hermano mayor de un hoy destacado y polémico jerifalte del Partido Popular.

\section{Apartado 3. Furia sexual.}

¡Qué tiempos! De enormes estragos sicalípticos pero también tiempos de claves. En el parte telefónico matinal de los lunes, facilitaba al Sabio el resultado de mis lances de fin de semana mediante la respuesta a dos preguntas: ¿Rossen? -por Robert Rossen, por El buscavidas, o sea qué había sucedido en el tapete verde- y ¿Miller? -por Henry Miller, por sus trópicos, o sea qué aventuras de alcoba había protagonizado-.

También la agenda (y vuelvo a cogerla) nos va a servir de ayuda. El domingo, 3 de marzo, entre otras informaciones, aparece el nombre Marisol seguido de dos flechas. La primera apunta a otro nombre - Jamborec (lo que entonces se denominaba una cava de jazz) - y a un dato estremecedor: Corri $3^{a}$ (tercera corrida - eyaculación, evidentemente, aunque no queda claro en este momento si el cómputo es diario y sólo referido a dicha Marisol-). La segunda flecha apunta a las siglas G.V., ya mencionadas antes, y que corresponden a Gran Vía, al piso situado en esa avenida, usado también para la organización de timbas, y, luego, aparece, como no podía ser de otra manera, Corri 4a. Pero lo inquietante viene ahora. Otra flecha, partiendo de G.V., termina en la siguiente frase: «Estamos pecando, lágrimas». 


\section{JORNADA LABORAL DE UN POETA BARCELONÉS}

Para no cansarles, sólo dos fechas más, y una consideración. 1. " fecha: sábado, 4 de mayo, un lacónico «Tarde G.V. Mary Gángster» que supone el primer paso en la carrera del poeta hacia el dominio de las artes ahogatorias que culminaría en el episodio titulado Rinola Cornejo y el estrangulador de Boston recogido en Papeles de Son Armadans. 2." fecha -no en esta agenda-: domingo, 29 de diciembre del mismo año 1963, «Chez-Nous. Mujer caballo. Faja» en la que solicito en el transcurso de un baile lento en la pista de la boite Chez-Nous que Carmen Ballesteros se despoje de la incómoda prenda.

La consideración, al hilo de lo contado, es de contenidos obvios: la maniobra preferida por el poeta, tanto por su rapidez como por su higiene, era el frotamiento, en sus dos variantes: la frontal o ventral que requería el pretexto del baile o de otras intimidades, y la posterior o cular, aún más agradable, ejercida en los transportes públicos pero no anónima, al fidelizar posturas, ubicaciones y, desde luego, cómplices.

Cerrando el apartado, algo excepcional. Un documento que se creía perdido y sobre el que incluso alguien, en un medio público, llegó a poner en duda su existencia. He de decir que yo mismo, a lo largo de tantas décadas, lo fui desfigurando y, en el instante de su hallazgo, ni la forma ni sus contenidos coincidieron con aquel folio y aquel título «Las doscientas mujeres más importantes de 1962» que de modo impenitente acudía mezclado a otras pesadillas en la rijosidad de mis sueños. Aquí está (lo muestro agitándolo), una hojita, un jirón del pasado, con un título estremecedor: «200 niñas relacionadas conmigo en los 2 últimos años (las más tratadas)» y, en un recuadro, la fecha $10-\mathrm{X}-60$.

En su mayoría, los nombres de las censadas, corresponden a apellidos que aún puedo relacionar (familias de Barcelona de toda la vida), pero luego hay un magma de motes de gran poder sugerente, algunos precedidos por signos cabalísticos, entre los que se cree descubrir, en algún caso, entre interrogantes, la palabra "casada". Estos apodos, agrupados quizá por nichos ecológicos, por gremios o por comarcas, constituyen bloques de cuatro o cinco unidades entre los que destaco por su aparente extravagancia los siguientes:

Dumbo Negro-King Creole-Gran Pestilencia-Abogado

Borracha-Galletas Patria-Meneítos-Toda Semen

Niña Niño-Niña Juncal-Niña Maña-Niña Muerta

Bestial Pasteles-Paralítica Ingrata-Sargento de San Antonio Voces-Mamante-¿Un Hombre?

(Sin saberse si esto último es un mote o una nota al anterior.)

\section{Apartado 4. Obra social.}

\section{Pedagogía.-}

Había que explicar el mundo. Y qué mejor manera que encuadrar las cosas en categorías. Y qué mejor que estas categorías fueran pocas. ¿Y si sólo fueran dos?

Eran estas: "Dios" y "Esputo"

La primera, por ejemplo, acogía a Orson Welles, a William Faulkner y a Piranesi.

La segunda, por ejemplo, acogía a Doris Day, a Gabriel Celaya y a la Jota Navarra.

La formulación era la siguiente - por ejemplo ante un texto-: "Esto es de Dios" o bien "Esto es un esputo".

\section{Dos curiosidades:}

1. ${ }^{\text {a- }}$ Teniendo en cuenta que el manejo de estas categorías, aunque no registrado, era de uso casi exclusivo de quien les habla y del poeta conocido por "el Sabio", habrá que reconocer la responsabilidad en que se incurría cada vez que ante las masas sedientas se daba un veredicto. 


\section{FRANCISCO FERRER LERÍN}

Por lo que no deja de ser sorprendente, desde la actual perspectiva, la inclusión, por parte de "el Sabio", en la categoría de Esputo, yo diría que en su grado máximo, de dos conceptos siempre peliagudos como son "lo religioso" y "lo catalán" aunque tal declaración se produjera ante un grupito de exaltados epígonos ávidos de noticias y en un clima de agradable relajación allá en la primavera de 1964.

2. a- Una declaración de principios que me encarga una profesora francesa radicada en Madrid para encabezar los cinco poemas que clla elige para una antología de la joven poesía española -hablo de 1970 - resulta ser una amplificación de las categorías citadas. Digo que: «Es fácil descubrir la génesis de mis poemas. El monstruo (1963), es un sueño puberal con Kafka y el Manual de zoología fantástica de Borges a medio digerir. Isabel de Herstad (1963), una abstracción modernista. La mano (1964), algo de fantaciencia, surrealismo y hemofobia. Se describe una vida extraña (1965), una abstracción del thriller. Tzara (1970), las anotaciones espontáneas a mi traducción de L'homme aproximatif». Y sigo: «Básicamente, esa fue mi poesía de 1960 a 1970. Se podrían añadir otras constantes: estructuración cinematográfica, literatura galante española, antirregionalismo, antichabacanería, Saint-John Perse, brujería, Joyce, dadaísmo, piratas, Freud, erotismo, Gabriel Miró, Henry Miller, Une saison en enfer, Whitman, y también la fauna silvestre europea, el póquer, las ciudades corrompidas..., y, en general, todo lo que está suficientemente sedimentado».

\section{Curaciones.-}

a) El Sabio, por ejemplo, lograba corregir los malos hábitos de los espectadores de las salas cinematográficas intensificando su habitual luminiscencia epidérmica. Recuerdo la proyección de L'Aventura en el cine Atenas de la calle Balmes de Barcelona, el año 63, en el ciclo Antonioni, cuando una pareja de novios empezó a toser de modo despiadado: nadie rechistaba, en parte porque era un filme con subtítulos, pero mi grado de incomodidad llegó hasta tal extremo que alargando el brazo rocé su hombro con la punta de mis dedos -siempre dejábamos una butaca libre entre los dos- y la señal, de modo instantáneo, produjo en el cuerpo del superdotado un destello de luz tan poderoso que los dos enfermos - sentados en la fila anterior- quedaron petrificados y, desde luego, curados de la tisis aguda neumónica que les afectaba desde hacía meses.

b) Sería a finales de curso del 70 ó del 71. Rinola Cornejo estaba esperándome sentada en un banco del claustro de la Universidad para darme la noticia. « $i$ Paco, Paco, me han diagnosticado un cáncer de cuello de matriz!» Aquel mismo día, sin relación aparente con su lesión, le pidió permiso a su marido, un alemán conocido por El solitario de Engadina, para irse conmigo a pasar el fin de semana en Jaca. Parece que le vea, en el rellano de su bloque del barrio obrero, preocupado por si Rinola se dejaba algo, ayudándonos a bajar el equipaje. Y luego, la avería, nos quedamos sin luz en los faros y tuvimos que hacer noche en Barbastro. El hotel estaba en ruinas, como la dueña, que resultó ser parienta lejana de mi abuela materna, y nuestra cama carecía de una pata, pero fuera por los ladrillos que la sustituían -lo que al moverse producía una extraña sensación acústica y dinámica- o por la peculiar especialidad amatoria de Rinola -el péndulo, ella encima en cuclillas, mi miembro circuncidado barriendo células malignas - lo cierto es que sanó a los pocos días. La criatura, agradecida, propagó por la facultad mis poderes curativos y tuve que soportar, a partir de aquel momento, un desfile de madres de condiscípulas prestas a embarcarse hacia el Somontano Oscense.

\section{Apartado 5. Producción artística.}

Tanta aventura dejó algunas obras aceptables, que se recogen en tres libros: De las condiciones humanas (1964), La hora oval (1971) y Cónsul (1987). Tras un paréntesis, cl poeta escribe un guión -Die Rabe (2001) - a instancias de Frederic Amat, artista plástico y cineasta. Se incluye completa la secuencia 031 de este documento. 


\section{JORNADA LABORAL DE UN POETA BARCELONÉS}

Interiores. Casino Pricipal de Jaca.

-Planos Generales (P.G.) cenitales y subjetivos de la sala de juego de la secuencia 007 (mirones espiando desde las falsas). Mismas tomas: conserje expulsando de la sala de juego a dichos mirones y única mesa de juego ocupada, sólo por dos jugadores, uno con los rasgos de I.erín y el otro de avanzada cdad y aspecto señorial.

-P.G. lateral de la mesa de juego. A cada lado de la pantalla un jugador: a la derecha Gran Lerín y a la izquierda Lerín, ambos inmóviles y mudos, vestidos de calle. No se ven mirones. Los murmullos de fondo van mitigándose. Al llegarse al silencio aparece por la parte izquicrda el conserje, por el fondo de la sala, que sin preámbulos toma asiento entre los dos jugadores, en el centro de la pantalla y de frente al espectador.

-Plano Medio (P.M.) lateral de la mesa de juego en ligero picado. Misma situación de los participantes. El conserje/croupier, de uniforme, en plena labor, baraja el mazo de cartas y lo deja a su derecha sobre el tapete. Mira a ambos jugadores y sin esperar respuesta da a cortar a Lerín. Vuelve a montar las cartas, coge la que ha quedado encima y la deja, tapada, en el centro del tapete.

-Primer Plano (P.P.) frontal del rostro del croupier. Dice, imperativamente: “¡Apuesten a ciegas!"

-P.M. lateral: Gran Lerín coge un billete de los que tiene de resto y lo echa al centro del tapete. Lerín, vacila unos instantes, pero iguala la cantidad echando también él un billete al centro. El croupier entonces da la vuelta a la carta dejándola descubierta en el centro del tapete.

-Plano de Detalle (P.D.) cenital de la carta situada en el centro del tapete: es el rey de copas de la baraja de póquer español (pertenece al modelo de naipes n² 20 de Heraclio Fournier).

-P.P. lateral de la cabeza de L.erín. Tiene la vista fija en la carta que se ha descubierto pero no se mueve, ni parpadea, ni mucho menos abre la boca.

-P.P. lateral de la cabeza de Gran I.erín. Misma situación.

-P.P. frontal del rostro del croupier. Vuelve a mirar a los dos jugadores, mira a la carta, y dice: "Comienza el juego. Obligatorio seguir el significado. Vence la última metáfora válida. Sólo antigua poesía germánica. La mano habla."; y gira el rostro hacia Lerín.

-P.P. frontal de Lerín que dejando de mirar la carta y mirando por primera vez a su adversario dice con aplomo: "Distribuidor de tesoros,"

-P.D. del naipe con la subtitulación "Distribuidor de tesoros = El Rey."

-P.P. frontal de Gran Lerín que, con seguridad, mirando a los ojos a Lerín, como recitando dice: "Distribuidor de espadas."

-P.D. del naipe con la subtitulación "Distribuidor de espadas = El Rey."

-P.P. frontal del croupier: "Primer envite nulo. Pueden aumentar las apuestas."

-P.M. lateral: ahora es Lerín quien toma la iniciativa y arroja dos billetes al centro; Gran Lerín, no demasiado entusiasmado, iguala la apuesta.

-P.P. frontal del croupier: “Ahora es mano aquí. Empiece.” Mira a Gran I.crín.

-P.P. frontal de Gran Lerín: "Piedras de la cara."; breve subtitulación: "Los ojos."

-P.P. frontal de L.erín: "Lunas de la frente."

-P.P. frontal del croupier: "Segundo envite nulo. Pueden aumentar las apuestas."

-P.M. lateral: Lerín echa dos billetes más y Gran Lerín, mecánicamente, iguala la apuesta. 


\section{FRANCISCO FERRER LERÍN}

-P.P. lateral de Lerín: "Hielo de la pelea"; aparece el subtítulo: "La espada."

-P.P. lateral de Gran Lerín: "Vara de la ira."

-P.M. lateral de la mesa: el croupier va a hablar -"Tercer envi..."- pero los contendientes se enzarzan en un duro combate: alternando, "Fuego de yelmos, Dragón encendido, Roedor de yelmos, Espina de la batalla, Pez de la batalla, Remo de la sangre, Lobo de las heridas, Rama de las heridas." De pronto callan. Se miran fijamente. El croupier parece incómodo, intenta recuperar su autoridad.

-P.P. frontal del croupier: "¡Señores, por favor, aténganse a las normas! Este envite queda invalidado. Y también esta carta. Voy a levanta otra. Pero antes apuesten si quieren."

-P.P. frontal de Gran Lerín. Está cansado. Mira su resto. Lo coge. Y arroja, sin contar, todos los billetes al centro de la mesa. Levanta los ojos y mira, con aire de fastidio, a Lerín.

-P.P. frontal de Lerín. Sorprendido, tarda algo en reaccionar pero también coge el dinero y lo lanza al centro,

-P.M. lateral: el croupier retira el Rey de Copas colocándolo bajo el mazo y coge la carta de encima, la voltea y la deja en el centro.

-P.D. cenital: el As de Oros.

-P.P. frontal del croupier. A Gran Lerín: "Usted es mano. Usted empicza."

-P.P. frontal del rostro de Gran Lerín que acusa enormemente el cansancio: "Peñasco de los hombros."

-P.D. cenital del As de Oros con el subtítulo: "Peñasco de los hombros = La cabeza."

-P.P. frontal del rostro de Lerín que denota confianza, casi alegría: "Castillo del cuerpo."

-P.M. lateral de la mesa: el croupier con voz impersonal, con cierto desprecio y ya con ánimo de terminar: "Si no van a incrementar las apuestas..."; la cámara se acerca a su rostro; "Si ya no hay restos..." "Quien termine con lo que yo les proponga se lo lleva todo"; medita unos segundos, mira a los dos; "Ya que es el As de Oros..." "Metáforas de Oro" "Aquí empieza”; y señala a Lerín.

-P.P. frontal del rostro de Lerín: mira a la carta, mira a Gran Lerín.

-P.D. frontal de parte del rostro de Lerín, de su boca que habla: "Lecho de la serpiente."

-P.P. frontal del rostro de Gran Lerín que parece como si ya no quisiera seguir -está fatigado pero sobre todo molesto-. Transcurren unos segundos y al final, como arrastrando las sílabas: "Resplandor de la mano."

-P.M. lateral de Gran Lerín: se levanta.

-P.M. frontal del croupier que, medio incorporado, y mirando a Lerín dice: “ ¡Esto no se ha acabado! ¡Vence quien termina! ¡Y usted puede seguir!

-P.P. frontal de Lerín, aún sentado, sonriente: “ßBronce de las discordias!”

-P.G. de la sala en la que se ve a Gran Lerín saliendo de la misma, al croupier junto a Lerín en actitud servil a la espera de propina y a Lerín abalanzándose sobre la mesa para coger el dinero.

-P.P. lateral en ligero picado de las manos de Lerín cogiendo los billetes.

\section{Epílogo.}

Como remate y para desintoxicarnos de tantas pequeñas heroicidades, aunque para abundar en la convicción de que todo texto ha de tener un sustrato de experiencia personal de cierto riesgo, me van a permitir que lea unas líneas de un artículo escrito por Ezra 


\section{JORNADA LABORAL DE UN POETA BARCELONÉS}

Pound y publicado en la revista Poetry en Junio de 1916. Es una traducción de 1970 hecha por José Vázquez Amaral, ni mejor ni peor que otras, para el editor mejicano Joaquín Mortiz.

El artículo se titula "La constante prédica a la chusma" y de él extraigo lo siguiente:

Una y otra vez la misma mentira. No tiene caso hablar de mentiras a los ignorantes, pues carecen de criterio. Algunos consideran que engañar a los ignorantes es una maldad, pero al demagogo le interesa apoyar su profesión y demostrar que la demagogia es la máxima obra de Dios. Y por eso leemos por milésima vez que la poesía se escribe para entretener. De la siguiente manera: «Los comienzos de la poesía inglesa... realizada por una gente guerrera y ruda para entretener a los hombres de armas, o para los hombres que se sentaban a la mesa de los monjes».

O estas afirmaciones se hacen para obtener los favores de otras personas sentadas frente a estériles mesas gordas, o se hacen por ignorancia que es charlatanería cuando pretende pasar por sagrado e impecable conocimiento.

«Los comienzos... para entretener»... ¿acaso ha leído quien afirma esto The Seafarer en anglosajón? ¿Nos quiere decir el autor para quién fueron escritas estas líneas, únicas entre las obras de nuestros antepasados que se pueden comparar con Homero..., para el entretenimiento de quién? No se hicieron para entretener a nadie, sino porque un hombre que se aferraba al silencio no pudo dejar de hablar. Y ese poema menos parejo, The Wanderer, se parece a aquel otro; en ambos habla un hombre destruido:

\section{Ne maeg werigmod wryde withstondan ne se hreo hyge helpe gefremman: for thon domgeorne dreorigne oft in hyra breostcofan bindath faeste.}

"For the doom-eager brindeth fast his blood-bedrag-gled heart in his breast»... una disculpa por atreverse a hablar, y el hablar sólo perdonado porque su capitán y todos los marinos y los compañeros han muerto; algunos muertos por los lobos, otros arrancados de los acantilados por las aves cuyos nidos saquearon.

Poemas como éstos no fueron escritos para los oradores de banquetes, como no lo es el undécimo libro de la Odisea. No obstante, la chusma se siente halagada cuando se le dice que su importancia es tan grande que el solaz de los hombres solitarios, y la más señorial de las artes, fue creada para su esparcimicnto.

Esta es la verdad.

Nada más. Muchas gracias.

Barcelona/Hix, primavera del 2002.

Ponencia leída en el congreso "Poéticas Novísimas".

Zaragoza, 27 de abril de 2002. Inédita, no se publicaron las actas. 